\title{
Some types of first order fractional differential equations and their applications
}

\author{
Chiihuei $\mathrm{Yu}^{*}$ \\ School of Mathematics and Statistics, Zhaoqing University, Guangdong, China
}

\begin{abstract}
Keywords: new multiplication, chain rule, modified Riemann-Liouville fractional derivatives, first order fractional differential equations.
\end{abstract}

\begin{abstract}
This paper uses a new multiplication of fractional functions and chain rule for fractional derivatives, regarding the Jumarie type of modified Riemann-Liouville fractional derivatives to obtain the general solutions of four types of first order fractional differential equations. On the other hand, some examples are proposed to illustrate our results.
\end{abstract}

\section{Introduction}

Fractional calculus is the field of mathematical analysis which deals with the investigation and applications of integrals and derivatives of arbitrary order. The fractional calculus, during the last years went through a great evolution [1-3]. The reason for the advancement and expansion is due to the great number of functions that fractional differentiation operators and integration provided to the academic community [4-7]. The fractional calculus may be considered an old and yet novel topic. It is an old topic since, starting from some speculations of Leibniz and Euler, it has been developed up to nowadays. A list of mathematicians, who have provided important contributions up to the middle of our century, includes Laplace, Fourier, Abel, Liouville, Riemann, Holmgren, Grünwald, Letnikov, Laurent, Nekrassov, Krug, Hadamard, Heaviside, Pincherle, Hardy and Littlewood, Weyl, L'evy, Marchaud, Davis, Zygmund, Love, Erd'elyi, Kober, Widder, Riesz.

In recent years, fractional calculus has been a fruitful field of research in science and engineering [7-10]. In fact, many scientific areas are currently paying attention to the fractional calculus concepts and we can refer its adoption in viscoelasticity and damping, diffusion and wave propagation, electromagnetism, chaos and fractals, heat transfer, electronics, signal processing, robotics, system identification, traffic systems, genetic algorithms, percolation, modelling and identification, telecommunications, physics, control systems, economy and finance. Unlike standard calculus, there is no unique definition of derivation and integration in fractional calculus. The commonly used definition is the Riemann-Liouville (R-L) fractional derivative [9]. Other useful definitions include Caputo definition of fractional derivative [11], the Grunwald-Letinikov (G-L) fractional derivative [9], and Jumarie's modified R-L fractional derivative [12].

\footnotetext{
*Corresponding author: 2019013010@zqu.edu.cn
} 
In this article, we study four types of first order fractional differential equations, regarding the Jumarie type of modified R-L fractional derivatives. A new multiplication of fractional functions is defined and we use chain rule for fractional derivatives to obtain the general solutions of these first order fractional differential equations. Moreover, some examples are given to demonstrate the advantage of our results.

\section{Preliminaries and methods}

Firstly, the fractional calculus used in this paper is introduced below.

Definition 2.1: Let $\alpha$ be a real number and $m$ be a positive integer. The modified Riemann-Liouville fractional derivatives of Jumarie type ([13]) is defined by

$$
{ }_{a} D_{x}^{\alpha}[f(x)]=\left\{\begin{array}{lc}
\frac{1}{\Gamma(-\alpha)} \int_{a}^{x}(x-\tau)^{-\alpha-1} f(\tau) d \tau, & \text { if } \alpha<0 \\
\frac{1}{\Gamma(1-\alpha)} \frac{d}{d x} \int_{a}^{x}(x-\tau)^{-\alpha}[f(\tau)-f(a)] d \tau & \text { if } 0 \leq \alpha<1 \\
\frac{d^{m}}{d x^{m}}\left({ }_{a} D_{x}^{\alpha-m}\right)[f(x)], & \text { if } m \leq \alpha<m+1
\end{array}\right.
$$

where $\Gamma(y)=\int_{0}^{\infty} t^{y-1} e^{-t} d t$ is the gamma function defined on $y>0$. If $\left({ }_{a} D_{x}^{\alpha}\right)^{n}[f(x)]=\left({ }_{a} D_{x}^{\alpha}\right)\left({ }_{a} D_{x}^{\alpha}\right) \cdots\left({ }_{a} D_{x}^{\alpha}\right)[f(x)]$ exists, then $f(x)$ is called $n$-th order $\alpha$-fractional differentiable function, and $\left({ }_{a} D_{x}^{\alpha}\right)^{n}[f(x)]$ is the $n$-th order $\alpha$-fractional derivative of $f(x)$. We note that $\left({ }_{a} D_{x}^{\alpha}\right)^{n} \neq{ }_{a} D_{x}^{n \alpha}$ in general. On the other hand, we define the fractional integral of $f(x),{ }_{a} I_{x}^{\alpha}[f(x)]={ }_{a} D_{x}^{-\alpha}[f(x)]$, where $\alpha>0$, and $f(x)$ is called $\alpha$-integral function. We have the following property [14].

Proposition 2.2: Let $\alpha, \beta, c$ be real numbers and $\beta \geq \alpha>0$, then

$$
{ }_{0} D_{x}^{\alpha}\left[x^{\beta}\right]=\frac{\Gamma(\beta+1)}{\Gamma(\beta-\alpha+1)} x^{\beta-\alpha},
$$

and

$$
{ }_{0} D_{x}^{\alpha}[c]=0 .
$$

Next, we introduce the fractional exponential function.

Definition 2.3 ([15]): The Mittag-Leffler function is defined by

$$
E_{\alpha}(z)=\sum_{k=0}^{\infty} \frac{z^{k}}{\Gamma(k \alpha+1)}
$$

where $\alpha$ is a real number, $\alpha>0$, and $z$ is a complex variable.

In the following, we introduce a new multiplication of fractional functions.

Definition 2.4 ([16]): Let $\lambda, \mu, z$ be complex numbers, $0<\alpha \leq 1, j, l, k$ be non-negative integers, and $a_{k}, b_{k}$ be real numbers, $p_{k}(z)=\frac{1}{\Gamma(k \alpha+1)} z^{k}$ for all $k$. The $\otimes$ multiplication is defined by

$$
\begin{aligned}
p_{j}\left(\lambda x^{\alpha}\right) \otimes p_{l}\left(\mu y^{\alpha}\right)= & \frac{1}{\Gamma(j \alpha+1)}\left(\lambda x^{\alpha}\right)^{j} \otimes \frac{1}{\Gamma(l \alpha+1)}\left(\mu y^{\alpha}\right)^{l}= \\
& \frac{1}{\Gamma((j+l) \alpha+1)}\left(\begin{array}{c}
j+l \\
j
\end{array}\right)\left(\lambda x^{\alpha}\right)^{j}\left(\mu y^{\alpha}\right)^{l},(5)
\end{aligned}
$$

where $\left(\begin{array}{c}j+l \\ j\end{array}\right)=\frac{(j+l) !}{j ! l !}$.

If $f_{\alpha}\left(\lambda x^{\alpha}\right)$ and $g_{\alpha}\left(\mu y^{\alpha}\right)$ are two fractional functions,

$$
\begin{aligned}
& f_{\alpha}\left(\lambda x^{\alpha}\right)=\sum_{k=0}^{\infty} a_{k} p_{k}\left(\lambda x^{\alpha}\right)=\sum_{k=0}^{\infty} \frac{a_{k}}{\Gamma(k \alpha+1)}\left(\lambda x^{\alpha}\right)^{k}, \\
& g_{\alpha}\left(\mu y^{\alpha}\right)=\sum_{k=0}^{\infty} b_{k} p_{k}\left(\mu y^{\alpha}\right)=\sum_{k=0}^{\infty} \frac{b_{k}}{\Gamma(k \alpha+1)}\left(\mu y^{\alpha}\right)^{k},
\end{aligned}
$$

then we define

$$
\begin{gathered}
f_{\alpha}\left(\lambda x^{\alpha}\right) \otimes g_{\alpha}\left(\mu y^{\alpha}\right)=\sum_{k=0}^{\infty} a_{k} p_{k}\left(\lambda x^{\alpha}\right) \otimes \sum_{k=0}^{\infty} b_{k} p_{k}\left(\mu y^{\alpha}\right) \\
=\sum_{k=0}^{\infty}\left(\sum_{m=0}^{k} a_{k-m} b_{m} p_{k-m}\left(\lambda x^{\alpha}\right) \otimes p_{m}\left(\mu y^{\alpha}\right)\right)
\end{gathered}
$$




\section{Proposition}

2.5: $f_{\alpha}\left(\lambda x^{\alpha}\right) \otimes g_{\alpha}\left(\mu y^{\alpha}\right)=\sum_{k=0}^{\infty} \frac{1}{\Gamma(k \alpha+1)} \sum_{m=0}^{k}\left(\begin{array}{c}k \\ m\end{array}\right) a_{k-m} b_{m}\left(\lambda x^{\alpha}\right)^{k-m}\left(\mu y^{\alpha}\right)^{m}$. (9)

Definition 2.6: Let $\left(f_{\alpha}\left(\lambda x^{\alpha}\right)\right)^{\otimes n}=f_{\alpha}\left(\lambda x^{\alpha}\right) \otimes \cdots \otimes f_{\alpha}\left(\lambda x^{\alpha}\right)$ be the $n$ times product of the fractional function $f_{\alpha}\left(\lambda x^{\alpha}\right)$. If $f_{\alpha}\left(\lambda x^{\alpha}\right) \otimes g_{\alpha}\left(\lambda x^{\alpha}\right)=1$, then $g_{\alpha}\left(\lambda x^{\alpha}\right)$ is called the $\otimes$ reciprocal of $f_{\alpha}\left(\lambda x^{\alpha}\right)$, and is denoted by $\left(f_{\alpha}\left(\lambda x^{\alpha}\right)\right)^{\otimes-1}$.

Definition 2.7: If $\mathrm{f}(z)=\sum_{k=0}^{\infty} a_{k} z^{k}, g_{\alpha}\left(\mu x^{\alpha}\right)=\sum_{k=0}^{\infty} b_{k} p_{k}\left(\mu x^{\alpha}\right)$, then

$$
f_{\otimes \alpha}\left(g_{\alpha}\left(\mu x^{\alpha}\right)\right)=\sum_{k=0}^{\infty} a_{k}\left(g_{\alpha}\left(\mu x^{\alpha}\right)\right)^{\otimes k}
$$

The following is the method we used in this paper.

Theorem 2.8 (chain rule for fractional derivatives) ([16]): Suppose that $f(z)=$ $\sum_{k=0}^{\infty} a_{k} z^{k} \quad, \quad g_{\alpha}\left(\mu x^{\alpha}\right)=\sum_{k=0}^{\infty} b_{k} p_{k}\left(\mu x^{\alpha}\right)$. If $f_{\otimes \alpha}\left(g_{\alpha}\left(\mu x^{\alpha}\right)\right)=$ $\sum_{k=0}^{\infty} a_{k}\left(g_{\alpha}\left(\mu x^{\alpha}\right)\right)^{\otimes k}$ and $f_{\otimes \alpha}^{\prime}\left(g_{\alpha}\left(\mu x^{\alpha}\right)\right)=\sum_{k=1}^{\infty} a_{k} k\left(g_{\alpha}\left(\mu x^{\alpha}\right)\right)^{\otimes(k-1)}$, then $\left({ }_{0} D_{x}^{\alpha}\right)\left[f_{\otimes \alpha}\left(g_{\alpha}\left(\mu x^{\alpha}\right)\right)\right]=f_{\otimes \alpha}^{\prime}\left(g_{\alpha}\left(\mu x^{\alpha}\right)\right) \otimes\left({ }_{0} D_{x}^{\alpha}\right)\left[g_{\alpha}\left(\mu x^{\alpha}\right)\right]$.

\section{Major results and discussions}

\subsection{The first order $\alpha$-fractional differential equation}

We solve Eq. (13) as follows:

$$
y=f\left(x,{ }_{0} D_{x}^{\alpha}[y]\right) \cdot(0<\alpha \leq 1)
$$

$$
\text { Let } \quad{ }_{0} D_{x}^{\alpha}[y]=p \quad \text {, then Eq. (13) becomes } y=f(x, p) \text {. }
$$

By chain rule for fractional derivatives, we have

$$
p={ }_{0} D_{x}^{\alpha}[y]={ }_{0} D_{x}^{\alpha}[f]+{ }_{0} D_{p}^{\alpha}[f] \otimes{ }_{0} D_{x}^{\alpha}[p] .
$$

Eq. (15) is a first order $\alpha$-fractional differential equation with respect to $x, p$.

Case 3.1.1 If the general solution of Eq. (14) is $p=\varphi(x, c)$, then $y=f(x, \varphi(x, c))$ is the general solution of Eq. (12).

Case 3.1.2 If the general solution of Eq. (14) is $x=\psi(p, c)$, then the general solution of Eq. (12) is the parametric equation form $\left\{\begin{array}{c}x=\psi(p, c) \\ y=f(\psi(p, c), p)\end{array}\right.$, where $p$ is a parameter and $c$ is any constant.

Case 3.1.3 If the general solution of Eq. (14) is $\Lambda(x, p, c)=0$, then we obtain the general solution of Eq. (12) is $\left\{\begin{array}{c}\Lambda(x, p, c)=0 \\ y=f(x, p)\end{array}\right.$, where $p$ is a parameter and $c$ is any constant. On the other hand, we note that fractional Clairaut's differential equation ([17]) is a special case of Eq. (12).

\subsection{Consider the first order $\alpha$-fractional differential equation}

$$
x=f\left(y,{ }_{0} D_{x}^{\alpha}[y]\right) \cdot(0<\alpha \leq 1)
$$

Let ${ }_{0} D_{x}^{\alpha}[y]=p$, then by chain rule for fractional derivatives, we obtain

$$
p^{\otimes-1}={ }_{0} D_{y}^{\alpha}[f]+{ }_{0} D_{p}^{\alpha}[f] \otimes{ }_{0} D_{y}^{\alpha}[p] .
$$

Since Eq. (16) is a first order $\alpha$-fractional differential equation with respect to $y, p$. If the general solution of Eq. (16) is $\Theta(y, p, c)=0$, then $\left\{\begin{array}{c}\Theta(y, p, c)=0 \\ x=f(y, p)\end{array}\right.$ is the general solution of Eq. (15). 


\subsection{Consider the first order $\alpha$-fractional differential equation}

$$
F\left(x,{ }_{0} D_{x}^{\alpha}[y]\right)=0 .(0<\alpha \leq 1)
$$

We also make ${ }_{0} D_{x}^{\alpha}[y]=p$, so $F(x, p)=0$. This equation represents a curve in the plane. If we express this curve as a parametric equation $\left\{\begin{array}{l}x=\varphi(t) \\ p=\psi(t)\end{array}\right.$, then by chain rule

It follows that

$$
p={ }_{0} D_{x}^{\alpha}[y]={ }_{0} D_{t}^{\alpha}[y] \otimes\left({ }_{0} D_{t}^{\alpha}[\varphi(t)]\right)^{\otimes-1} .
$$

$$
{ }_{0} D_{t}^{\alpha}[y]={ }_{0} D_{t}^{\alpha}[\varphi(t)] \otimes \psi(t) .
$$

Therefore, we obtain the general solution of Eq. (17) is the following parametric equation

$$
\left\{\begin{array}{c}
x=\varphi(t) \\
y={ }_{0}{ }_{t}^{\alpha}\left[{ }_{0} D_{t}^{\alpha}[\varphi(t)] \otimes \psi(t)\right]+c
\end{array}\right.
$$

\subsection{The first order $\alpha$-fractional differential equation}

$$
F\left(y,{ }_{0} D_{x}^{\alpha}[y]\right)=0 .(0<\alpha \leq 1)
$$

Let $p={ }_{0} D_{x}^{\alpha}[y]$, then $F(y, p)=0$. This equation represents a plane curve. If this curve can express as a parametric equation $\left\{\begin{array}{l}y=\varphi(s) \\ p=\psi(s)\end{array}\right.$, then using chain rule yields

It follows that

$$
\psi(t)={ }_{0} D_{x}^{\alpha}[y]={ }_{0} D_{s}^{\alpha}[\varphi(s)] \otimes\left({ }_{0} D_{s}^{\alpha}[x]\right)^{\otimes-1} .
$$

$$
{ }_{0} D_{s}^{\alpha}[x]={ }_{0} D_{s}^{\alpha}[\varphi(s)] \otimes(\psi(s))^{\otimes-1} .
$$

Thus the general solution of Eq. (21) is a parametric equation

$$
\left\{\begin{array}{c}
x={ }_{0} I_{s}^{\alpha}\left[{ }_{0} D_{s}^{\alpha}[\varphi(s)] \otimes(\psi(s))^{\otimes-1}\right]+c \\
y=\varphi(s)
\end{array}\right.
$$

\section{Applications}

For the four types of first order fractional differential equations discussed in this paper, some examples are provided and we find their general solutions.

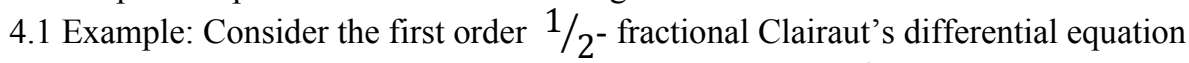

$$
y=\frac{1}{\Gamma\left(\frac{3}{2}\right)} x^{1 / 2} \otimes{ }_{0} D_{x}^{1 / 2}[y]+3\left({ }_{0} D_{x}^{1 / 2}[y]\right)^{\otimes 2} .
$$

Let $p={ }_{0} D_{x}^{1 / 2}[y]$, then

$$
y=\frac{1}{\Gamma\left(\frac{3}{2}\right)} x^{1 / 2} \otimes p+3 p^{2}
$$

Seeking the fractional derivative of $x$ on both sides of Eq. (26) yields

So,

$$
p=p+\frac{1}{\Gamma\left(\frac{3}{2}\right)} x^{1 / 2} \otimes{ }_{0} D_{x}^{1 / 2}[p]+6 p \otimes{ }_{0} D_{x}^{1 / 2}[p] .
$$

$$
\left(\frac{1}{\Gamma\left(\frac{3}{2}\right)} x^{1 / 2}+6 p\right) \otimes{ }_{0} D_{x}^{1 / 2}[p]=0
$$


If ${ }_{0} D_{x}^{1 / 2}[p]=0$, then $p=c$ is a constant. Thus, $y=\frac{c}{\Gamma\left(\frac{3}{2}\right)} x^{1 / 2}+3 c^{2}$. If $\frac{1}{\Gamma\left(\frac{3}{2}\right)} x^{1 / 2}+$ $6 p=0$, then $x^{1 / 2}=-6 \Gamma\left(\frac{3}{2}\right) p$, and hence $x=36\left(\Gamma\left(\frac{3}{2}\right)\right)^{2} p^{2}$. So, the general solution of Eq. (25) is the parameter equation

$$
\left\{\begin{array}{c}
x=36\left(\Gamma\left(\frac{3}{2}\right)\right)^{2} p^{2} \\
y=-6 p c+3 c^{2}
\end{array}\right.
$$

Example 4.2: The first order $1 / 3$ - fractional differential equation

$$
{ }_{0} D_{x}^{1 / 3}[y]=\left(y^{1 / 3}+\Gamma\left(\frac{4}{3}\right)\right) \otimes x .
$$

Since Eq. (30) is separable, it follows from [18] that it has the general solution

$$
y^{1 / 3}=c \cdot E_{1 / 3}\left(\frac{1}{\Gamma\left(\frac{7}{3}\right)} x^{4 / 3}\right)-\Gamma\left(\frac{4}{3}\right)
$$

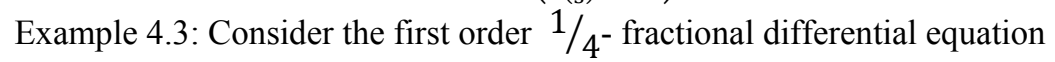

$$
\left({ }_{0} D_{x}^{1 / 4}[y]\right)^{\otimes 3}=\frac{1}{\Gamma\left(\frac{3}{2}\right)} x^{1 / 2}+\frac{2}{\Gamma\left(\frac{5}{4}\right)} x^{1 / 4}+3 .
$$

Let $x^{1 / 4}=t$, i.e., $x=t^{4}$. If $p={ }_{0} D_{x}^{1 / 4}[y]$, then $p^{3}=\frac{1}{\Gamma\left(\frac{3}{2}\right)} t^{2}+\frac{2}{\Gamma\left(\frac{5}{4}\right)} t+3$. Therefore, $p={ }_{0} D_{x}^{1 / 4}[y]=\Gamma\left(\frac{5}{4}\right) \cdot{ }_{0} D_{t}^{1 / 4}[y]$. It follows that ${ }_{0} D_{t}^{1 / 4}[y]=\frac{1}{\Gamma\left(\frac{5}{4}\right)} p=\frac{1}{\Gamma\left(\frac{5}{4}\right)}\left(\frac{1}{\Gamma\left(\frac{3}{2}\right)} t^{2}+\right.$ $\left.\frac{2}{\Gamma\left(\frac{5}{4}\right)} t+3\right)^{1 / 3}$.

And hence, $y={ }_{0} I_{t}^{1 / 4}\left[\frac{1}{\Gamma\left(\frac{5}{4}\right)}\left(\frac{1}{\Gamma\left(\frac{3}{2}\right)} t^{2}+\frac{2}{\Gamma\left(\frac{5}{4}\right)} t+3\right)^{1 / 3}\right]+c$. Therefore, the general solution of Eq. (32) is the parametric equation

$$
\left\{y={ }_{0} I_{t}^{1 / 4}\left[\frac{1}{\Gamma\left(\frac{5}{4}\right)}\left(\frac{1}{\Gamma\left(\frac{3}{2}\right)} t^{2}+\frac{2}{\Gamma\left(\frac{5}{4}\right)} t+3\right)^{1 / 3}\right]+c .\right.
$$

Example 4.4: The first order $1 / 5^{-}$fractional differential equation

$$
\left({ }_{0} D_{x}^{1 / 5}[y]\right)^{\otimes 7}=\frac{1}{\Gamma\left(\frac{9}{5}\right)} y^{4 / 5}-\frac{3}{\Gamma\left(\frac{8}{5}\right)} y^{3 / 5}+\frac{4}{\Gamma\left(\frac{7}{5}\right)} y^{2 / 5}-6 E_{1 / 5}\left(\frac{1}{\Gamma\left(\frac{6}{5}\right)} y^{1 / 5}\right) .
$$

If $y^{1 / 5}=s$, i.e., $y=s^{5}$. Let $p={ }_{0} D_{x}^{1 / 5}[y]$, then

$$
p^{7}=\frac{1}{\Gamma\left(\frac{9}{5}\right)} s^{4}-\frac{3}{\Gamma\left(\frac{8}{5}\right)} s^{3}+\frac{4}{\Gamma\left(\frac{7}{5}\right)} s^{2}-6 E_{1 / 5}\left(\frac{1}{\Gamma\left(\frac{6}{5}\right)} s\right) \text {. }
$$

So, $\frac{1}{p}={ }_{0} D_{y}^{1 / 5}[x]=\Gamma\left(\frac{6}{5}\right) \cdot{ }_{0} D_{s}^{1 / 5}[x]$. It follows that

$$
{ }_{0} D_{s}^{1 / 5}[x]=\frac{1}{\Gamma\left(\frac{6}{5}\right)} \cdot \frac{1}{p}=\frac{1}{\Gamma\left(\frac{6}{5}\right)} \cdot \frac{1}{\left(\frac{1}{\Gamma\left(\frac{9}{5}\right)} s^{4}-\frac{3}{\Gamma\left(\frac{8}{5}\right)} s^{3}+\frac{4}{\Gamma\left(\frac{7}{5}\right)} s^{2}-6 E_{1 / 5}\left(\frac{1}{\Gamma\left(\frac{6}{5}\right)}\right)\right)^{1 / 7}} .
$$

And hence, 


$$
x={ }_{0} I_{s}^{1 / 5}\left[\frac{1}{\Gamma\left(\frac{6}{5}\right)} \cdot \frac{1}{\left(\frac{1}{\Gamma\left(\frac{9}{5}\right)} s^{4}-\frac{3}{\Gamma\left(\frac{8}{5}\right)} s^{3}+\frac{4}{\Gamma\left(\frac{7}{5}\right)} s^{2}-6 E_{1 / 5}\left(\frac{1}{\Gamma\left(\frac{6}{5}\right)} s\right)\right)^{1 / 7}}\right]+c .
$$

Thus, the general solution of Eq. (34) is the parametric equation

$$
\left\{x={ }_{0} I_{s}^{1 / 5}\left[\frac{1}{\Gamma\left(\frac{6}{5}\right)} \cdot \frac{1}{\left(\frac{1}{\Gamma\left(\frac{9}{5}\right)} s^{4}-\frac{3}{\Gamma\left(\frac{8}{5}\right)} s^{3}+\frac{4}{\Gamma\left(\frac{7}{5}\right)} s^{2}-6 E_{1} / 5\left(\frac{1}{\Gamma\left(\frac{6}{5}\right)} s\right)\right)^{1 / 7}}\right]+c .\right.
$$

\section{Conclusions}

We can see from the above discussion, the general solutions of four types of first order fractional differential equations studied in this article can be obtained mainly using a new multiplication and chain rule for fractional derivatives. In fact, the applications of the two methods are extensive, and can be used to easily solve many fractional differential equations. In fact, our results are generalizations of classical first order differential equations. In the future, we will use the Jumarie type of modified R-L fractional derivatives and the new multiplication to expand our research into the problems of engineering mathematics and fractional calculus.

\section{References}

1. R. Herrmann, Fractional Calculus: An Introduction for Physicists, World Scientific Publishing Company, Singapore, (2011).

2. A. A. Kilbas, H. M. Srivastava, J. J. Trujillo, Theory and Applications of Fractional Differential Equations, North-Holland Mathematics Studies, Elsevier, Amsterdam, Vol. 207, (2006).

3. S. G. Samko, A. A. Kilbas, O. I. Marichev, Fractional Integrals and Derivatives, Theory and Functions, Gordon and Breach, Yverdon, (1993).

4. S. S. Ray, Fractional Calculus with Applications for Nuclear Reactor Dynamics, CRC Press, Boca Raton, Fla., USA, (2015).

5. K. Oldham, J. Spanier, The Fractional Calculus. Theory and Applications of Differentiation and Integration to Arbitrary Order, 111, Academic Press, New York/London, (1974).

6. R. L. Magin, Fractional calculus in bioengineering, in: 2012 13th International Carpathian Control Conference, ICCC 2012, (2012).

7. F. Mainardi, Fractional Calculus and Waves in Linear Viscoelasticity: An Introduction to Mathematical Models, World Scientific, (2010).

8. A, Oustaloup, LaDérivation Non Entière: Théorie, Synthèse et Applications, Editions Hermès, Paris, (1995).

9. I. Podlubny, Fractional Differential Equations, Academic, San Diego, (1999). 
10. K. S. Miller, B. Ross, An Introduction to the Fractional Calculus and Fractional Differential Equations, Wiley, New York, (1993).

11. S. Das, Functional Fractional Calculus, 2nd ed. Springer-Verlag, (2011).

12. G. Jumarie, Modified Riemann-Liouville derivative and fractional Taylor series of nondifferentiable functions further results, Computers \& Mathematics with Applications, Vol. 51, No. 9, (2006), pp.1367-1376.

13. D. Kumar, J. Daiya, Linear fractional non-homogeneous differential equations with Jumarie fractional derivative, Journal of Chemical, Biological and Physical Sciences, Vol. 6, No. 2, (2016), pp. 607-618.

14. U. Ghosh, S. Sengupta, S. Sarkar, and S. Das, Analytic solution of linear fractional differential equation with Jumarie derivative in term of Mittag-Leffler function, American Journal of Mathematical Analysis, Vol. 3, No. 2, (2015), pp.32-38.

15. J. C. Prajapati, Certain properties of Mittag-Leffler function with argument $x^{\alpha}, \alpha>0$, Italian Journal of Pure and Applied Mathematics, Vol. 30, (2013), pp. 411-416.

16. C. -H. Yu, Differential properties of fractional functions, International Journal of Novel Research in Interdisciplinary Studies, Vol.7, No. 5, (2020), pp.1-14.

17. C. - H. Yu, Fractional Clairaut's differential equation and its application, International Journal of Computer Science and Information Technology Research, Vol. 8, Issue 4, (2020), pp. 46-49.

18. C. -H. Yu, Separable fractional differential equations, International Journal of Mathematics and Physical Sciences Research, Vol. 8, Issue 2, (2020), pp. 30-34. 